\title{
Process Control in a Press Hardening Production Line with Numerous Process Variables and Quality Criteria
}

\author{
Anke Stoll, Norbert Pierschel, Ken Wenzel, and Tino Langer \\ Fraunhofer Institute for Machine Tools and Forming Technology IWU, \\ Reichenhainer Str. 88, 09126 Chemnitz, Germany \\ anke.stoll@iwu.fraunhofer.de
}

\begin{abstract}
Today, the optimization of the press hardening process is still a complex and challenging task. This report describes the combination of linear regression with least squares optimization to adjust the process parameters of this process for quality improvement. The FE simulation program AutoForm was used to model the production line concerned and various process and quality parameters were measured. The proposed system is capable of automatically adjusting the process parameters of following process steps based on the quality estimate at each step of the production line. An additional benefit is the identification of likely defective parts early in the production process. Based on the results derived from 1000 observations a better understanding of the process was obtained and in the future the combined regression and optimization approach can be extended to more complex production lines.
\end{abstract}

Keywords: linear regression, least squares optimization, production line, press hardening, process control

\section{Introduction}

One of the goals of Industry 4.0 is the optimization and customization of production processes through digitization with algorithms, big data approaches and high technologies [1]. Currently, machine learning (ML) approaches support monitoring, diagnosis and (off-line) system optimization for fault detection, maintenance, decision support and product quality improvement $[2,3]$. The field of ML is manifold and various different methods are available. However, in manufacturing and other fields of application the complexity of ML methods can hinder their adoption even though the data acquisition for many production processes is possible and a sufficient data base is available or can be obtained. Therefore, this work aims to implement a simplistic ML and optimization approach for a production line. The paper starts with a discussion of work related to ML and process control in Section 2, followed by the presentation of the methodology in Section 3, that includes a description of the data sets, the data preparation, and the estimation techniques. The results of the analysis are described in Section 4. Section 5 presents the conclusions and discussion of practical implications. 


\section{State of the Art}

First approaches for process control based on ML were conducted by $\mathrm{Oh}$ and co-workers [4] who apply Neural Network/Partial Least Squares to model the relationship between multiple process parameters and multiple quality parameters in the production process of metal plates of a complex structure. Senn and co-workers [5] use Principal Component Analysis and Artificial Neural Networks to model the relation between observed quantities and state variables for a deep drawing process. However, comprehensive studies for ML based process control within production lines are still sparse. In order to contribute to fill this gap we propose an intuitive approach to intelligently control the process parameters within a production line for quality improvement of the final product. The introduced intelligent system is based on linear regression and least squares optimization.

\section{$3 \quad$ Data and Methods}

We consider a production line for the press hardening of sheet metal in order to produce center pillars, which are ultra-high-strength car body parts. Here, we will focus on the three process steps warming, handling and quenching, see Figure 1. The process involves inserting sheets, which have been heated beyond the austenitizing temperature of about $900^{\circ} \mathrm{C}$, into a cooled forming tool, in which they are then quenched. The thermal integrated processing produces presshardened parts with an extremely high tensile strength of up to 1,500 $\mathrm{MPa}$ for the ultra-high-strength steel $22 \mathrm{MnB} 5$. The handling of the sheets is done by robots.

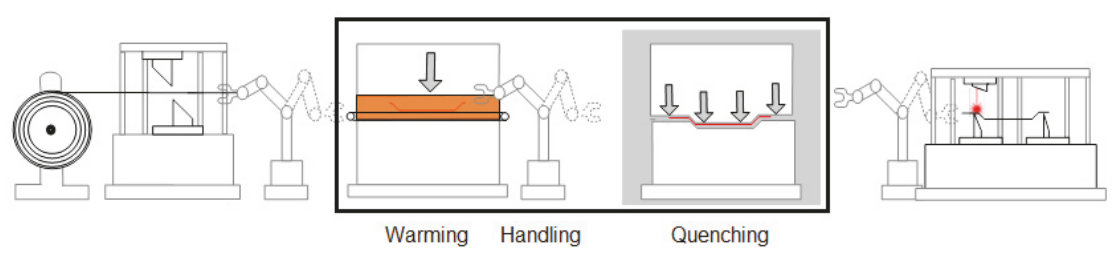

Fig. 1. Production line for the press hardening of sheet metal focusing on the three steps: (1) warming in a furnace unit, (2) handling with a robot system with grippers, and (3) quenching.

Similar to Oh et al. [4] each process can be described by its

- uncontrollable factors (initial conditions of materials or processes; and fix variables),

- controllable factors (adjustable variables) and

- quality variables (response variables representing the final product quality). 
Figure 2 shows the parameters we considered in our case study. Uncontrollable input variables are the sheet thickness (ST) and the tool temperature during quenching (ToTemp). Controllable input variables are sheet temperature after warming (STemp), transfer time between warming and quenching (TT), quenching force $(\mathrm{QF})$, quenching time (QT) and spacing (Sp). Quality variables are the output variables hardness at a critical point $\mathrm{P} 1$ on the finished part $(\mathrm{P} 1 \mathrm{H})$ and sheet thickness at another critical point P2 (P2ST). The ML method proposed in the next section then correlates input and output variables and allows process intervention for quality improvement. Data were acquired using the sheet metal forming software AutoForm [6], similar to [7]. The whole data set consists of 1000 observations which were achieved by variation of the input parameters as shown in Table 1.

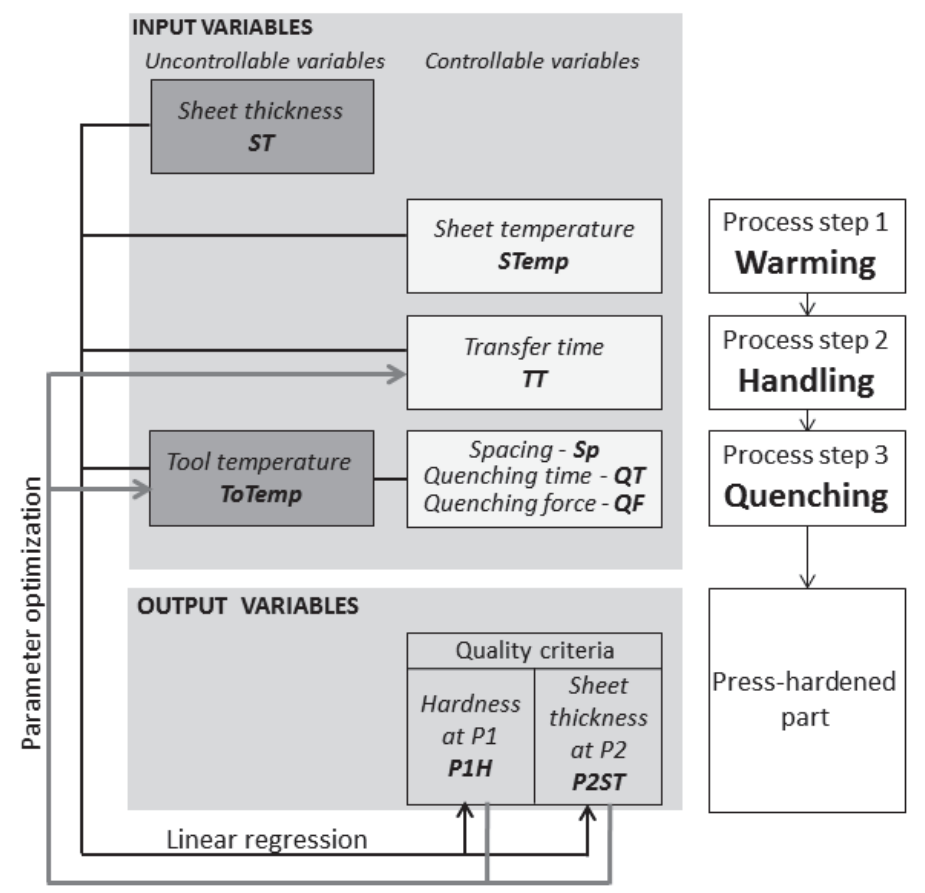

Fig. 2. Production line with three process steps and their respective controllable and uncontrollable variables. Linear regression is conducted based on the existing database. After the warming process is finished, parameter optimization for the process steps handling and quenching is possible.

\subsection{Data Preparation}

The open source statistical programming tool $\mathrm{R}[8,9]$ was used to evaluate the data generated by AutoForm. The aim of this study was to find an appropriate 
ML model to describe the relationship between the input and output parameters. Upper and lower boundaries for the allowed input parameter variations are defined as stated in Table 1. Boundaries for the quality criteria have to be defined as well. These depend on the type of component that is produced. The focus can be on maximum component hardness or for example on the maximum thickness of the finished component. As we focus on a part from the automotive industry we want to maximize/increase both, the sheet thickness and hardness at critical points which are prone to tearing. Thus, no upper boundaries for $\mathrm{P} 1 \mathrm{H}$ and P2ST were defined.

Table 1. Process parameters, quality criteria, and regression coefficients for the estimation of $\mathrm{P} 1 \mathrm{H}$ and P2ST.

\begin{tabular}{lrrr||rr|r} 
Variable & $\begin{array}{c}\text { lower } \\
\text { boundary boundary }\end{array}$ & $\begin{array}{c}\text { upper default } \\
\text { P1H }\end{array}$ & $\begin{array}{c}\text { coefficients } \\
\text { P2ST }\end{array}$ \\
\hline ST $[\mathrm{mm}]$ & 1.45 & 1.55 & 1.5 & - & $9.4 \times 10^{-1}$ \\
STemp $\left[{ }^{\circ} \mathrm{C}\right]$ & 900 & 950 & 900 & 1.1 & - \\
TT [s] & 5 & 55 & 5 & -6.2 & $4.9 \times 10^{-4}$ \\
ToTemp $\left[{ }^{\circ} \mathrm{C}\right]$ & 80 & 300 & 190 & $-7.4 \times 10^{-2}$ & $-5.3 \times 10^{-6}$ \\
QF [kN] & 500 & 2,500 & 2,000 & $3.5 \times 10^{-6}$ & $-1.3 \times 10^{-9}$ \\
QT [s] & 2 & 65 & 2 & $3.3 \times 10^{-1}$ & $-1.2 \times 10^{-4}$ \\
Sp [mm] & 0.1 & 2 & 1.05 & 1.1 & $2.4 \times 10^{-3}$ \\
Intercept & & & & $-4.5 \times 10^{2}$ & $6.2 \times 10^{-2}$ \\
\hline P1H [HV] & 390 & & & & \\
P2ST [mm] & 1.43 & & & & \\
\hline
\end{tabular}

\subsection{Linear Regression for Quality Prognosis}

Description of the Model Ultimately, we aim for on-line process control which makes the application of high speed models and fast predictions necessary. As a first step - conducted off-line - we need to describe the relationship between input and output variables in a distinguishable way. A general linear model which accounts for the single parameters linear effects was considered. In general, a linear regression equation has the following form

$$
\text { DepVar }=a+\left(b_{1} \times \operatorname{Indep}_{\operatorname{Var}_{1}}\right)+\ldots+\left(b_{n} \times \operatorname{Indep}_{\operatorname{Var}_{n}}\right) .
$$

Where $a, b_{1}, \ldots, b_{n}$ are unknown parameters, DepVar stands for dependent variable and represents the qualities $\mathrm{P} 1 \mathrm{H}$ and P2ST, respectively. IndepVar's are the independent variables, such as the process parameters.

The analysis is carried out in R using the $\operatorname{lm}()$ function for fitting linear models independently for the two quality parameters $\mathrm{P} 1 \mathrm{H}$ and P2ST. The resulting regression coefficients are shown in Table 1. 
Validation of the Model The regression analysis indicates that STemp, TT, ToTemp, QF, QT and Sp had significant influence on $\mathrm{P} 1 \mathrm{H}$, which is confirmed by the $p$-values (no significant influence of ST). The overall suitability of a linear regression approach is supported by an adjusted $R^{2}$ of 0.90 which describes the percentage of the dependent variable variation by the model. P2ST can be thoroughly described by linear combinations of ST, TT, ToTemp, QF, QT and Sp (no significant influence of STemp) with an adjusted $R^{2}$ of 0.99 .

Since the total number of observations is limited and a partition into training and test data is not sensible without loosing significant modeling capability the models were validated with $K$-fold cross-validation. For $K=5$, the overall mean square of prediction error is 97.6 for the linear model (compared to 102 for the complete model with all variables) to predict $\mathrm{P} 1 \mathrm{H}$ and $3.87 \times 10^{-6}$ for the prediction of P2ST (compared to $6.14 \times 10^{-6}$ for the complete model). This indicates reasonably good linear models despite the limited number of observations which will be increased in the future.

\subsection{Least Squares Optimization with Constraints}

Set-up of the Optimization Problem After each step in the production line the qualities $\mathrm{P} 1 \mathrm{H}$ and $\mathrm{P} 2 \mathrm{ST}$ are estimated using the variables already measured in combination with assumptions for variables of the process steps not yet performed (default values in Table 1). These assumptions are based on technological expert knowledge. After the warming process, we know the ST and the STemp. In order to get a first estimate for the expected quality $\mathrm{P} 1 \mathrm{H}$ and $\mathrm{P} 2 \mathrm{ST}$ we use the linear regression model established in 3.2 with the measured ST and STemp and default values for TT, ToTemp, Sp, QT and QF as stated in Table 2. If the estimated quality is below the predefined threshold, also stated in Table 2, the controllable variables in following process steps have to be adjusted in order to bring the quality back into its desirable interval. An optimization process was established, which calculates the necessary adjustments. Least squares are applied to solve the emerging inhomogeneous linear system with constraints after every process step. With each step the accuracy of the model improves as less and less process estimates have to be used to predict the quality.

In order to solve the optimization problem least squares with equality and inequality constraints is performed. The function from the R-package limSolve is called lsei() and solves

$$
\min \|A x-b\| \text { subject to } E x=f, G x \geq h .
$$

For the optimization after process step 1 (warming) the matrix $A$ is the unity matrix with dimension 4 because there are four subsequently determinable variables left in the manufacturing process. The vector $b$ contains the default values / desirable process values for the 4 adjustable variables. The objective function tries to find a solution for the 4 adjustable variables which is as close as possible to the desired default values. Since our optimization problem does not have equalities, $E$ is a zero matrix of the dimension 4 and $f$ is a vector of zeros. The inequality constraint $G x \geq h$ is constructed from the upper and 
lower boundaries of the adjustable variables and the linear regression equations combined with the quality boundaries. The optimization after process step 2 is conducted in a similar way but only 4 adjustable variables are remaining.

Weighting of Parameters Since an adjustment of some parameters is easier than others, e.g. TT or QT, weighted least squares can be used to improve the efficiency of parameter optimization. The weighting vector $W_{a}$ as an additional input for the lsei() optimization function is defined to prefer changes on easily adjustable variables such as TT and QT. Thus the weighting coefficients for TT and QT were chosen 1 while they are 100 for QF and Sp. By giving each variable its proper amount of influence on the resulting quality a more realistic image of the real press hardening process is established. The weight for each variable is given relative to the weights of the other variables.

\section{Results and Discussion}

In order to show the versatility of the approach four different scenarios are presented in the following.

The type of component to be produced has an immediate impact on the optimization problem. The system can be optimized towards the hardness of the produced component, process velocity (usually as fast as possible to be costeffective), geometric accuracy or other objectives. In the production industry the overall equipment effectiveness $(\mathrm{OEE})$ is a relevant and popular indicator for a machine or production line. Thus, we want to focus on a process as fast as possible which correlates directly with the maximization of the number of cycles in a production line. For this purpose, the default setting for TT is the smallest possible value of $5 \mathrm{~s}$, similar to a minimum QT of $2 \mathrm{~s}$. The QF has to be as high as possible in order to allow the quenching process to be fast. Thus, a QF of $2,000 \mathrm{kN}$ is chosen as default allowing slight upward adjustment with a total maximum of $2,500 \mathrm{kN}$. The Sp default is $1.05 \mathrm{~mm}$.

The quality control of the production line can return an "accepted part" for parts meeting both quality criteria as defined in Table 1 and "defective part" otherwise.

\subsection{No Parameter Adjustment Necessary}

In the majority of cases a production line should produce high quality parts when working with feasible process parameter intervals. One example for a process cycle resulting in an accepted part is shown in Table 2 . The warming process is conducted with a ST of $1.5 \mathrm{~mm}$ and a resulting STemp of $900^{\circ} \mathrm{C}$. Both $\mathrm{P} 1 \mathrm{H}$ and $\mathrm{P} 2 \mathrm{ST}$ are estimated with the linear regression approach described in section 3.2 with default values for TT, QF, QT and Sp (see Table 1). The predicted P1H and P2ST imply a qualitatively accepted part. Even with a longer than targeted TT of $10 \mathrm{~s}$ instead of $5 \mathrm{~s}$ the quality at the end of the process is still within range (Table 2, row 3 ) and no parameter adjustment is necessary (Table 2, row 4). 
Table 2. No adjustment necessary. Highlighted in gray are the process parameters already known.

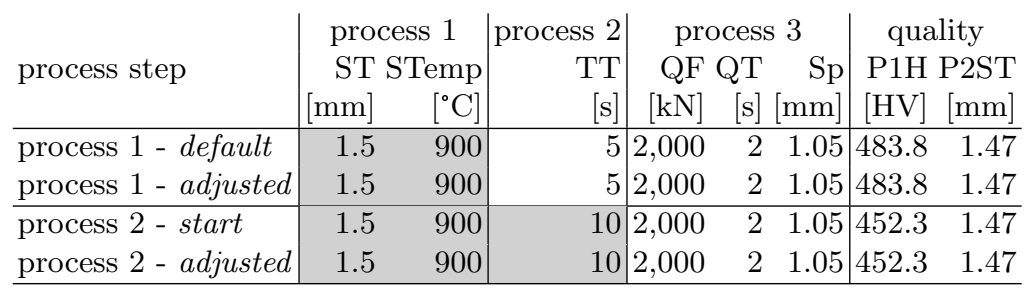

\subsection{Parameter Adjustment}

If the ST is $1.45 \mathrm{~mm}$ instead of $1.5 \mathrm{~mm}$ with an identical STemp of $900^{\circ} \mathrm{C}$ the estimated P2ST is too low. If the process is not adjusted, this cycle will likely produce a rejected part. However, the proposed approach allows an adjustment of the parameters in process step 2 and 3 in order to produce an accepted part. The model suggests a TT of $19.8 \mathrm{~s}$ instead of $5 \mathrm{~s}$, a maximum QF of $2,500 \mathrm{kN}$ and a slightly increased $\mathrm{Sp}$ of $1.2 \mathrm{~mm}$ in order to obtain a part with the required sheet thickness (Table 3, row 2). If the suggested TT of $19.8 \mathrm{~s}$ is slightly longer with $20.5 \mathrm{~s}, \mathrm{P} 1 \mathrm{H}$ is outside the feasible interval and an adjustment in process 3 is necessary (Table 3, row 3). Here, the QT is increased to $12.8 \mathrm{~s}$ and the Sp is increased to $1.71 \mathrm{~mm}$ in order to obtain a part with accepted quality (Tabe 3 , row 4$)$.

Table 3. Parameter adjustment. Written in bold are violated quality criteria.

\begin{tabular}{l|rr|rrr|rrr} 
& \multicolumn{3}{|c|}{ process 1 } & process 2 & \multicolumn{2}{|c|}{ process 3 } & \multicolumn{2}{c}{ quality } \\
process step & \multicolumn{2}{|c|}{ ST STemp } & TT & QF & QT & Sp & P1H P2ST \\
& {$[\mathrm{mm}]$} & {$\left[{ }^{\circ} \mathrm{C}\right]$} & {$[\mathrm{s}]$} & {$[\mathrm{kN}]$} & {$[\mathrm{s}]$} & {$[\mathrm{mm}]$} & {$[\mathrm{HV}]$} & {$[\mathrm{mm}]$} \\
\hline process 1 - default & 1.45 & 900 & 5 & 2,000 & 2 & 1.05 & 480.8 & $\mathbf{1 . 4 2}$ \\
process 1 - adjusted & 1.45 & 900 & 19.8 & 2,500 & 2 & 1.2 & 390.0 & 1.43 \\
\hline process 2 - start & 1.45 & 900 & 20.5 & 2,500 & 2 & 1.2 & $\mathbf{3 8 5 . 9}$ & 1.43 \\
process 2 - adjusted & 1.45 & 900 & 20.5 & 2,500 & 12.8 & 1.71 & 390.0 & 1.43 \\
\hline
\end{tabular}

\subsection{Limited Adjustment}

For an even lower sheet thickness of $1.44 \mathrm{~mm}$ and STemp of $900^{\circ} \mathrm{C}$ the quality criterion P2ST is violated with $1.41 \mathrm{~mm}$ instead of $1.43 \mathrm{~mm}$. An adjustment of the process parameters of process 2 and 3 is not possible without violating some of the constraints as the optimization approach aims for keeping both quality criteria within their intervals and at the same time all process parameters within their boundaries. Thus, a limited adjustment is performed in order to obtain a part as close as possible to accepted quality (Table 4, row 2). The parameters of process 2 and 3 are altered such that $\mathrm{P} 1 \mathrm{H}$ is just at the lower limit and P2ST 
is improved as much as possible $(1.423 \mathrm{~mm}$ instead of $1.41 \mathrm{~mm}$ and close to accepted quality). For this purpose, TT is increased from $5 \mathrm{~s}$ to $19.8 \mathrm{~s}, \mathrm{QF}$ is at its maximum and $\mathrm{Sp}$ is increased to $1.99 \mathrm{~mm}$.

Table 4. Limited adjustment / no adjustment possible. Again, violated quality criteria are written in bold. Marked with a star are improved by still violated qualities.

\begin{tabular}{|c|c|c|c|c|c|c|c|}
\hline \multirow{3}{*}{ process step } & \multicolumn{3}{|c|}{ process 1 process 2} & \multicolumn{3}{|c|}{ process 3} & \multirow{3}{*}{$\begin{array}{c}\text { quality } \\
\text { P1H P2ST } \\
\text { [HV] } \quad[\mathrm{mm}]\end{array}$} \\
\hline & \multicolumn{2}{|c|}{ ST STemp } & \multirow{2}{*}{$\begin{array}{r}\mathrm{TT} \\
{[\mathrm{s}]}\end{array}$} & \multicolumn{3}{|c|}{ QF QT $\quad$ Sp } & \\
\hline & {$[\mathrm{mm}$} & {$\left[{ }^{\circ} \mathrm{C}\right]$} & & {$[\mathrm{kN}]$} & s & [mm] & \\
\hline process 1 - default & 1.44 & 900 & 5 & 2,000 & 2 & 1.05 & $480.2 \quad \mathbf{1 . 4 1}$ \\
\hline process 1 - adjusted & 1.44 & 900 & 19.8 & 2,500 & 2 & 1.99 & $390.01 .423^{*}$ \\
\hline process 2 - start & 1.45 & 900 & 20.5 & 2,500 & 2 & 1.99 & 385.41 .423 \\
\hline process 2 - adjusted & 1.45 & 900 & 20.5 & 2,500 & 2 & 1.99 & 385.41 .423 \\
\hline
\end{tabular}

\subsection{No Adjustment Possible}

Sometimes the quality prognosis after process step 1 indicates that the produced part will not meet the final product quality requirements. Given the fact, that the prognosis is accurate, this is a very valuable information this early on in a production line because defective parts can be removed early in the production process with the additional benefit of cost and energy savings. Table 4 shows an example where after process step 2 no parameter adjustment is possible without violating the constraints. HP1 and P2ST will both be too low no matter how the process parameters in process 3 are altered.

\section{Discussion and Conclusions}

A combination of linear regression and least squares optimization can be employed to reproduce a bidirectional relation between process parameters and quality parameters in a fast and reliable manner. The proposed system is capable of estimating the quality outcome at any step of a production line. It allows adjustment of the controllable variables one or more process steps further on and identifies defective parts early in the production process.

If more than one quality criterion is considered, conflicting relations between them have to be expected. The goal of the parameter optimization is that parameter adjustments are found such that all quality criteria are satisfied. This constraint may result in an unsolvable optimization problem. The unsolvability of the problem after the first production step (or later in the process) indicates that the final product might not satisfy at least one quality criterion. The quality prognosis this early on in the production process is a valuable information, as potentially defective parts can be sorted out early. This saves resources, machine time and energy. 
The accuracy of the quality prognosis is mainly driven by the accuracy of the regression model. Therefore, a sufficiently large database is necessary. In the future, we plan to increase the data volume for a higher prognosis accuracy. The simulated data should be as close as possible to reality. Typically, the parameters of the press-hardening cycles follow a normal distribution and most of these cycles produce accepted parts. However, in the AutoForm software, a mesh is placed over the boundaries of the process parameters and the parameter variations are evenly distributed over the mesh. How this affects the regression model remains to be investigated. A validation of the ML approach with FEM simulations is under way. Once the extended simulation based regression and optimization approach works we will move on to experimental data and other more complex production lines.

Acknowledgments. This work was supported by the Fraunhofer-Gesellschaft with the funding of the lead project "ML4P - Machine Learning 4 Production". Furthermore we thank the European Union, the Free State of Saxony as well as the Fraunhofer-Gesellschaft for the funding of the High Performance Center Smart Production. Many thanks to Thomas Lieber for acquiring the simulation data.

\section{References}

1. Lu, Y.: Industry 4.0: a survey on technologies, applications and open research issues. Journal of Industrial Information Integration 6, 1-10 (2017)

2. Harding, J.A., Shahbaz, M., Kusiak, A.: Data mining in manufacturing: a review. Journal of Manufacturing Science and Engineering 128.4, 969-976 (2006)

3. Niggemann, O., Stein, B., Maier, A.: Solving Modeling Problems with Machine Learning A Classification Scheme of Model Learning Approaches for Technical Systems. In Model-Based Development of Embedded Systems (MBEES), Dagstuhl (2012)

4. Oh, S., Han, J., Cho, H.: Intelligent process control system for quality improvement by data mining in the process industry. Data mining for design and manufacturing. Springer US, 289-309 (2001)

5. Senn, M., Link, N.: A universal model for hidden state observation in adaptive process controls. International Journal on Advances in Intelligent Systems 4(3-4), $245-255(2012)$

6. AutoForm, url: https://www.autoform.com/

7. Neugebauer, R., Schieck, F., Polster, S., Mosel, A., Rautenstrauch, A., Schönherr, J., Pierschel, N.: Press hardening An innovative and challenging technology. Archives of civil and mechanical engineering 12(2), 113-118 (2012)

8. Ihaka, R., Gentleman, R.: R: A language for data analysis and graphics. Journal of Computational and Graphical Statistics 5(3), 299 - 314 (1996)

9. R Core Team: R: A Language and Environment for Statistical Computing. R Foundation for Statistical Computing, Vienna, Austria, https://www.R-project.org/ (2017) 
Open Access This chapter is licensed under the terms of the Creative Commons Attribution 4.0 International License (http://creativecommons.org/licenses/by/4.0/), which permits use, sharing, adaptation, distribution and reproduction in any medium or format, as long as you give appropriate credit to the original author(s) and the source, provide a link to the Creative Commons licence and indicate if changes were made

The images or other third party material in this chapter are included in the chapter's Creative Commons licence, unless indicated otherwise in a credit line to the material. If material is not included in the chapter's Creative Commons licence and your intended use is not permitted by statutory regulation or exceeds the permitted use, you will need to obtain permission directly from the copyright holder.

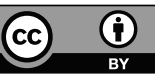

